\section{Fabrication of irregular optical phased arrays on silicon-on-insulator wafers}

\author{
Feng Xiao, Guangyuan Li, Yanping Li, and Anshi Xu \\ Peking University, School of Electronics Engineering \\ and Computer Science, State Key Laboratory of Advanced \\ Optical Communication Systems \& Networks, Beijing, \\ 100871, China \\ E-mail: lyrxas@pku.edu.cn
}

\begin{abstract}
A 16- and a 32-element irregular optical phased array based on silicon-on-insulator materials were fabricated. Such a device consists of an array of irregularly spaced single-mode waveguides, each of which has a specific length to give an appropriate phase retardation controllable by the wavelength. The optical beam can be steered continuously in the range of about 0 to 3 deg, corresponding to the wavelength varying from 1550.7 to $1551.9 \mathrm{~nm}$. () 2008 Society of Photo-Optical Instrumentation Engineers. [DOI: 10.1117/1.2902475]
\end{abstract}

Subject terms: optical phased array; irregular array; optical beam steering; phased array.

Paper 070982 L received Dec. 15, 2007; accepted for publication Feb. 3, 2008; published online May 1, 2008.

\section{Introduction}

Optical phased arrays provide an elegant means for the inertialess, high-resolution random-access beam steering that is required by numerous applications. As direct functional analogs of their microwave precursors, optical phased arrays have attracted worldwide attention for their considerable importance in the optical domain. ${ }^{1-7}$ Much research work has been performed on bulk modulators, ${ }^{8,9}$ integrated optics, ${ }^{3,4}$ nematic liquid crystal, ${ }^{1}$ and lead lanthanum zirconium titanate. ${ }^{10}$ However, due to the orders-of-magnitude difference in wavelengths between the microwave and optical worlds, optical phased arrays are difficult to implement. One main difficulty is that large phase-shifter spacings seem unavoidable, so that maximum steering angles are limited and efficiencies are low due to the large number of grating lobes.

The concept of irregular phased arrays has been introduced into the optics domain, ${ }^{11}$ in order to overcome the obstacle that elements have to be spaced sufficiently closely $(d \leqslant \lambda$, where $d$ is the interelement spacing and $\lambda$ is the optical wavelength ${ }^{11}$ ) to eliminate the grating lobes in the far-field radiation pattern. According to the present state of the technology, the method of irregular phased arrays can be adopted to achieve efficient large-angle beam steering in spite of large-size phase modulators and large interelement spacings. In order to overcome the problem of making $d \leqslant \lambda$ and simultaneously reduce the complexity of electrically addressing the vast number of phase modulators required by a high-performance optical phased array, a wavelength-controlled irregular optical phased array was

0091-3286/2008/\$25.00 @ 2008 SPIE proposed by the authors. ${ }^{6}$ It was shown that the irregular optical phased array (IOPA) can provide a large steering angle through a relatively simple device, in spite of large center-to-center interelement spacings. This configuration can be considered as an extension of a conventional arrayed waveguide grating (AWG) where waveguides are uniformly spaced. ${ }^{12}$

Some theoretical work on IOPAs has been reported. ${ }^{6,7,11,13}$ However, corresponding experiments, to the best of our knowledge, have received little effort. In this letter, we report an experimental investigation of wavelength-controlled irregular optical phased arrays based on silicon-on-insulator (SOI) wafers. Continuous deflections of about 3 deg have been observed while the wavelength varied from 1550.7 to $1551.9 \mathrm{~nm}$.

\section{Design and Fabrication}

The theory of wavelength-controlled irregular phased arrays is well described in Ref. 6, and the main results are given in the following. The interelement spacing $d_{r}$ and the optical path difference $n_{c} \Delta l_{r}\left(\Delta l_{r}=l_{r+1}-l_{r}\right)$ between adjacent waveguides should satisfy

$n_{c} \Delta l_{r}=m_{r} \lambda_{0}$,

$n_{s} d_{r}=D \cdot m_{r} \cdot \Delta \lambda_{\max }$,

and then constructive interference occurs only in the direction of the so-called main lobe,

$\theta_{\text {main }}=\arcsin \left(\frac{\Delta \lambda}{D \Delta \lambda_{\text {max }}}\right)$.

Here $l_{r}$ is the length of the $r^{\prime}$ th arrayed waveguide, $n_{c}$ is the effective refractive index of the waveguides, $n_{s}$ is the refractive index of the radiation zone, $m_{r}$ is the $r^{\prime}$ th grating order, $\lambda_{0}$ is the central wavelength, $\Delta \lambda=\lambda_{0}-\lambda, \Delta \lambda_{\max }$ is half of the operating wavelength range (i.e., the optical wavelength $\lambda$ varies from $\lambda_{0}-\Delta \lambda_{\max }$ to $\left.\lambda_{0}+\Delta \lambda_{\max }\right), r$ $=1,2, \ldots, N-1, N$ is the number of arrayed waveguides, and $D$ is a proportionality coefficient determining the dispersion capability of the phased array and the maximum deflection angle.

The device was designed using fundamental parameters as follows: the central wavelength $\lambda_{0}=1.55 \mu \mathrm{m}$; half of the operating wavelength range, $\Delta \lambda_{\max }=0.01 \mu \mathrm{m} ; n_{s}=1$; the number of arrayed waveguides, $N=16$ or 32 ; and the dispersion coefficient $D=2$. A set of grating orders $m_{r}$ was randomly selected in an appropriate range to determine the interelement spacing $d_{r}$ and the adjacent length difference $\Delta l_{r}$ to construct the device.

The layouts for the two IOPAs corresponding to 32 and 16 elements, respectively, are shown in Fig. 1, which is a unit taken from the mask for photoetching. All the waveguides in these devices are single-mode rib waveguides. The light is launched into the device by the input waveguide (IW). The Rowland circle structure, widely used in AWGs for free-propagation regions (FPRs), is introduced to split the input light and excite the arrayed waveguides (AWs) with same phase distribution. It is noted that the front end of the AWs is uniformly spaced even though their back end is nonuniform, as shown in the inset 


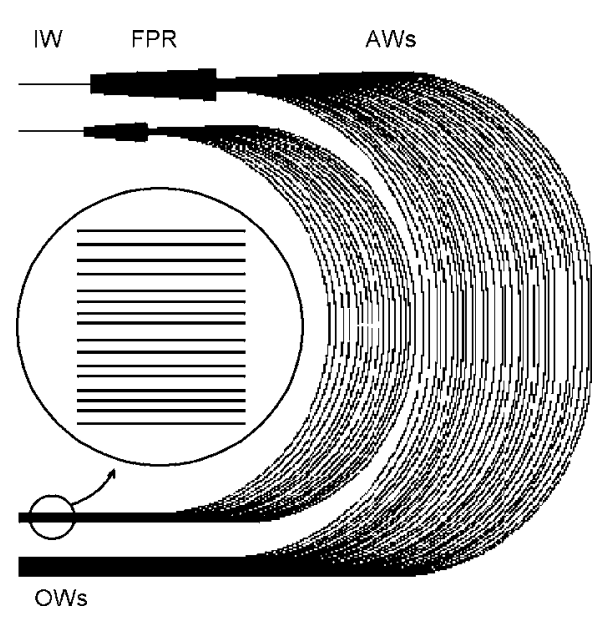

Fig. 1 A unit from the mask. IW: input waveguide; FPR: freepropagation region; AWs: arrayed waveguides; OWs: output waveguides. The inset shows the enlarged OWs of the 16-element IOPA.

of Fig. 1. The function of the AWs is to provide an appropriate phase retardation system by which the desired phase slope resulting in optical beam steering is formed. This phase slope can be controlled by the wavelengths. After propagation through the AWs, the light is coupled into the parallel output waveguides (OWs), which are spaced nonuniformly from 12 to $24 \mu \mathrm{m}$ according to the design parameters $d_{r}$

The designed devices were fabricated on a SOI wafer having a $4.9-\mu \mathrm{m}$-thick $\mathrm{Si}$ layer on top of a $0.4-\mu \mathrm{m}$-thick $\mathrm{SiO}_{2}$ layer, using the standard integrated optics process. The main fabrication process is inductively coupled plasma etching. The fabricated devices are shown in Fig. 2, where the input and output ends were polished to reduce the coupling loss, which is important in the following test.

\section{Test and Results}

After the fabrication, testing was performed using the setup shown in Fig. 3. Through an optical fiber fixed on a highprecision 3-D adjuster, a tunable laser light was coupled into the device, which was put on an $X Y Z$ flexure stage with high-precision manual adjusters. After propagation in the IOPA, the light is diffracted. A silver-coated mirror was placed behind the OWs to turn the optical field $90 \mathrm{deg}$ down so as to avoid the 3-D high-precision adjuster where the optical fiber was placed. A lens was followed to obtain the Fraunhofer diffraction pattern, which was captured by a CCD camera situated in the focal plane of the lens. Each CCD photograph corresponds to a wavelength.

A 16-element IOPA was tested, and the results are shown in Fig. 4. Each graph corresponds to a wavelength.

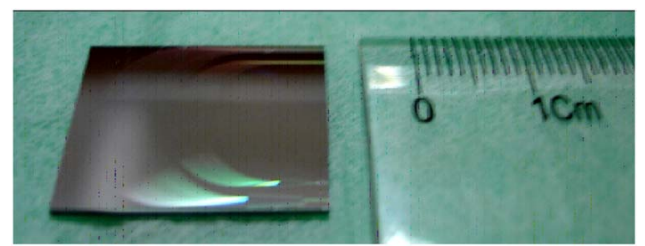

Fig. 2 Fabricated devices.

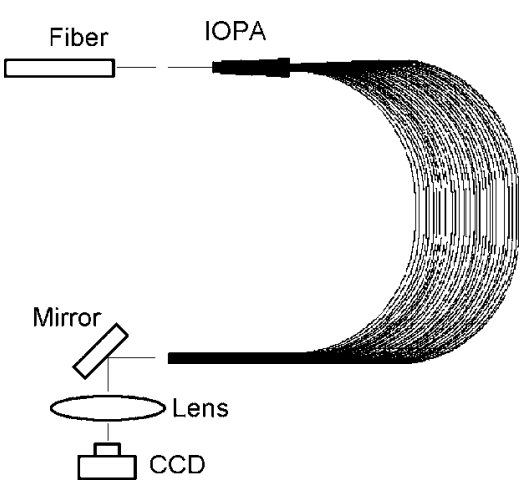

Fig. 3 Test setup.

The optical beam can be steered continuously in the range of about 0 to $3 \mathrm{deg}$ by changing the wavelength from 1550.7 to $1551.9 \mathrm{~nm}$. However, when the main lobe is steered out of that range, it is difficult to distinguish the main lobe from sidelobes, due to high sidelobe levels. This may be caused by random errors in the fabrication process that result in an imperfect phase slope as a result of the fluctuations in the optical path lengths of AWs. We roughly measured the beam width (FWHM), which is about $0.3 \mathrm{deg}$ and agrees well with the theoretical value. It is disappointing that the performance of the 32-element IOPA is no better than that of the 16-element one except that the beam width is about $0.25 \mathrm{deg}$. The reason for that may lie in two factors. One is that the 32-element IOPA occupies a larger area on the wafer, and consequently larger phase errors in the optical path lengths of AWs may be produced and deteriorate the performance. The other is that a few waveguides in the 32-element IOPA were broken during the fabrication, so that this IOPA has less than 32 elements. We did not measure the waveguide loss accurately; it was estimated to be greater than $5 \mathrm{~dB} / \mathrm{cm}$ due to sidewall roughness.

Figure 5 shows a comparison between the calculated results and the experimental data. These two results, having almost the same slope, indicate that the dispersion capability of the fabricated IOPA agrees well with the design. There is a parallel displacement of about 2.5 deg between them, which does not affect the performance and can be

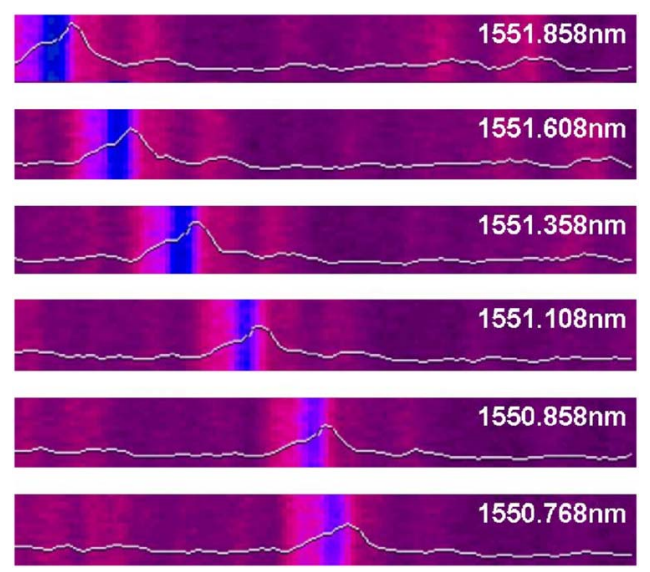

Fig. 4 Deflection angle versus optical wavelength. 


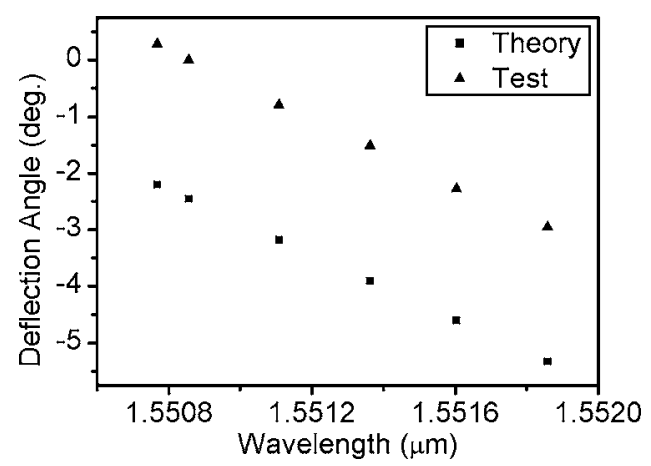

Fig. 5 Experimental results versus theoretical calculation.

compensated by adjusting the central wavelength $\lambda_{0}$. This is definitely caused by system errors in the process of fabrication, while the random errors will lead to performance degeneration.

\section{Conclusions}

As an initial attempt, an irregular optical phased array was fabricated. The devices consist of an array of 16 or 32 irregularly spaced single-mode rib waveguides, each of which has a specific length to give an appropriate phase retardation controllable by wavelengths. The optical beam can be continuously steered from about 0 to 3 deg by varying the wavelength from 1550.7 to $1551.9 \mathrm{~nm}$. A more precise mask and fabrication process are necessary to achieve a high-performance IOPA, which should be further investigated. This device has potential applications in deflectors, gratings, optical filters, multiplexers and demultiplexers, etc.

\section{Acknowledgments}

This work is supported by the National Natural Science Foundation of China under grant No. 60772002.

\section{References}

1. P. F. McManamon, T. A. Dorschner D. C. Corkum, L. S. Friedman, D. S. Hobbs, M. Holz, S. Liberman, H. Q. Nguyen, D. P. Resler, R. C. Sharp, and E. A. Wastson, "Optical phased array technology," Proc. IEEE 84, 268-298 (1996).

2. J. A. Thomas and Y. Fainman, "Optimal cascade operation of optical phased-array beam deflectors," Appl. Opt. 37, 6196-6212 (1998).

3. D. R. Wight, J. M. Heaton, B. T. Hughes, J. C. H. Birbeck, K. P. Hilton, and D. J. Taylor, "Novel phased array optical scanning device implemented using GaAs/AlGaAs technology," Appl. Phys. Lett. 59, 899-901 (1991).

4. F. Vasey, F. K. Reinhart, R. Houdre, and J. M. Stauffer, "Spatial optical beam steering with an AlGaAs integrated phased array," Appl. Opt. 32, 3220-3232 (1993).

5. M. K. Smit, "New focusing and dispersive planar component based on an optical phased-array," Electron. Lett. 24, 385-386 (1988).

6. F. Xiao, W. W. Hu, and A. S. Xu, "Optical phased-array beam steering controlled by wavelength," Appl. Opt. 44, 5429-5433 (2005).

7. S. Yin, J. H. Kim, F. Wu, P. Ruffin, and F. Luo, "Ultra-fast speed, low grating lobe optical beam steering using unequally spaced phased array technique," Opt. Commun. 270, 41-46 (2007).

8. R. A. Meyer, "Optical beam steering using a multichannel lithium tantalate crystal," Appl. Opt. 11, 613-616 (1972).

9. Y. Ninomiya, "Ultrahigh resolving electrooptic prism array light deflectors," IEEE J. Quantum Electron. QE-9(8), 791-795 (1973).

10. J. A. Thomas and Y. Fainman, "Programmable diffractive optical element using a multichannel lanthanum-modified lead zirconate titanate phase modulator," Opt. Lett. 20(13), 1510-1512 (1995).

11. J. H. Abeles and R. J. Deri, "Suppression of sidelobes in the far-field radiation patterns of optical waveguide arrays," Appl. Phys. Lett. 53 , 1375-1377 (1988)

12. F. Xiao, G. Y. Li, and A. S. Xu, "Modeling and design of irregularly arrayed waveguide gratings," Opt. Express 15, 3888-3901 (2007).

13. T. Y. Zhu, "Irregular virtual optical phased-array," Proc. SPIE 3951, 61-71 (2000). 